\title{
The effect of the use of a physical-activity mobile application on body composition and sleep quality of overweight children
}

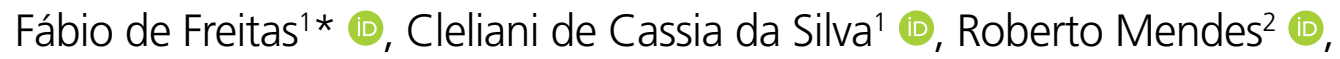 \\ Maria Ângela Antonio² (1), Mariana Zambon²
}

\begin{abstract}
SUMMARY
OBJECTIVE: To analyze the use of a mobile physical activity application and its influence on body composition and sleep quality in overweight children.

METHODS: Prospective study with 37 patients from the Child and Adolescent Obesity Clinic at Unicamp Hospital das Clínicas, between 2018 and 2019. Anamnesis and anthropometry were performed. We oriented the use of the application for six weeks, five days/week for ten minutes.

RESULTS: Among 37 patients, 28 (mean age 10.7 \pm 2.0 years, 50\% boys) used the application. The average use of the application ranged from $1.93 \pm 2.18$ to $3.25 \pm 1.84$ times/week, with a peak in the second week and a progressive decrease during follow-up. The paired $\mathrm{t}$-test showed, on average, lean mass (t (27)=-2.91), weight (t (27)=-3.11) and height ( $\mathrm{t}(27)=-3.79)$. After using the application, these were higher than before (all $p<0.05)$. There was a significant difference in the proportion of children who presented difficulty sleeping $(\chi 2(1)=5.143)$ and insomnia $\left(\chi^{2}(1)=4.167\right)$.

CONCLUSIONS: There was an improvement in sleep quality and an increase in lean mass, but no significant changes in BMI z-score, waist circumference, and body fat percentage.

KEYWORDS: Physical activity. Obesity. Child. Mobile application.
\end{abstract}

\section{INTRODUCTION}

Prevention and control of obesity among children and adolescents present a challenge to healthcare professionals as they involve improvement in dietary behavior, physical activity, and sleep quality. New technologies, such as mobile health applications, have emerged as an aid in this process ${ }^{1}$. These applications allow users to customize their goals and plans, and to switch between activities ${ }^{2}$. Most patients can benefit from these services, even if they live in remote communities and have limited access to places that provide care, because they are reliable, accurate, and inexpensive ${ }^{3}$.

Mobile-health applications present an effective and sustainable solution that can help users promote significant and consistent changes in their behavior towards an active and healthy lifestyle ${ }^{3}$. Studies indicate that they offer a fun alternative for users to improve their physical condition, concentration, and

\footnotetext{
'Universidade Estadual de Campinas, Faculdade de Ciências Médicas, Pós-Graduação em Saúde da Criança e do Adolescente - Campinas (SP), Brazil. ${ }^{2}$ Universidade Estadual de Campinas, Faculdade de Ciências Médicas, Departamento de Pediatria - Campinas (SP), Brazil.

*Corresponding author: fabio.edf.7@gmail.com

Conflicts of interest: the authors declare there is no conflicts of interest. Funding: this study was financed in part by the Coordenação de Aperfeiçoamento de Pessoal de Nível Superior - Brasil (CAPES) - Finance Code 001.

Received on December 11, 2020. Accepted on December 28, 2020.
} 
sleep. These applications can motivate users to play sports, and can be used outdoors as well as in groups ${ }^{4,5}$.

Given the current concerns regarding weight-management and increased screen time among children, this study aimed at evaluating the effect the use of a mobile application, by promoting physical activity, might have on the body composition and sleep quality of overweight children.

\section{METHODS}

This prospective, interventional study was conducted between February 2018 and August 2019, and included male and female patients who were in follow-up care at the Child and Adolescent Obesity Clinic of the Unicamp Hospital das Clínicas. Ten new cases of children between the ages of two and seventeen were admitted to the clinic every month.

Cases that met the inclusion criteria were selected. We selected 37 patients between the ages of seven and thirteen who had a suitable smartphone, were able to understand the application's functionality and the exercises, and were either overweight or obese. Patients signed an Informed Consent or a Minor Informed Consent form. Among the exclusion criteria were the presence of former psychiatric disorders, other concomitant illnesses such as endocrine, renal, pulmonary, or cardiovascular diseases, and girls who had started menstruating.

For the intervention, we used the Tabata Timer $^{\circledR}$ application for physical activity, and instructed patients to use the application at least five days a week, for six consecutive weeks. This application is available for free on several platforms, and it helps users to structure and modify the intensity of their exercises, supporting short-duration workout sessions. We helped patients set up the application, the time settings, and structured the proposed activities. We selected the following age-appropriate motor coordination and strength exercises, which were performed sequentially in timed intervals: jumping jacks; stationary single-leg hop exercises (hopscotch); squats; and the plank. The activities were structured as follows (with time set in seconds): 10 s preparation - 20s jumping jacks - 10s pause - 20s stationary single-leg hop exercises - 10s pauset - 20s squats - 10s pause - 20s plank - 50s pause to end the cycle. This sequence was performed in a set of four cycles, totaling ten minutes a day. During this period, the researcher and the children's parents communicated via WhatsApp Messenger ${ }^{\circledR}$ to receive a brief report of the activities. Right after each session, the Borg Rating of Perceived Exertion ${ }^{6}$ scale was presented: $1-2$ easy, $3-5$ moderate, and $\geq 6$ very difficult. We took anthropometric (physical) and bioimpedance (body-composition) measurements at the beginning and at the end of the six-week period. We assessed sleep quality before and after patients used the application by asking the following questions:

1) Do you have trouble sleeping? and

2) Do you have or have ever had insomnia? We considered sleep time to be the reported in the number of hours between sleep onset and wakefulness.

We took weight and height measurements ${ }^{7}$ and calculated BMI and BMI z-scores for age and gender, in order to classify the subjects as being overweight or obese ${ }^{8}$. We also measured their waist circumference (WC) ${ }^{9}$ and used tetrapolar bioimpedance with the Bioimpedance Analyzer - BIA $310^{\circledR 10}$.

For statistical analysis, we used the SPSS ${ }^{\circledR}$ program, version 16 , with a significance level of $\mathrm{p}<0.05$. We used the ShapiroWilk test to assess normality, and the paired t-test or McNemar's test to compare the variables.

\section{RESULTS}

Seven among the 37 patients did not use the application at any time, and two patients were excluded due to irregular adherence and drop-out. As a result, we examined 28 patients.

The average age was $10.7 \pm 2.0$ years ( $11.0 \pm 1.9$ for males and $10.4 \pm 2.2$ for females), with 2 overweight and 26 obese children.

Figure 1 illustrates the patients' average use of the application and the Borg Rating over six weeks.

Table 1 presents a comparison of the body composition and total sleep time before and after the subjects used the application. The paired t-test showed that lean mass $(t(27)=-2.91$, $\mathrm{p}=0.007)$, weight $(\mathrm{t}(27)=-3.11, \mathrm{p}=0.004)$, and height $(\mathrm{t}(27)=-$ $3.79, \mathrm{p}=0.001$ ) were statistically higher after the children used the application. BMI Z-scores $(\mathrm{t}(2)=0.678)$, WC $(\mathrm{t}(27)=0.465)$, body fat percentage $(t(27)=1.04)$, and the total sleep time $(t(27)=1.14)$ did not present statistically significant differences.

In Table 2, the exact McNemar test demonstrated a significant difference in the proportion of children who had trouble sleeping $(\chi 2(1)=5.143 ; \mathrm{p}=0.016)$ and those who had insomnia $(\chi 2(1)=4.167 ; \mathrm{p}=0.03)$ before and after using the application.

\section{DISCUSSION}

In this study, we assessed the effect that the use of a physical-activity mobile application has on the body composition and sleep quality of overweight children. There was little compliance with the recommended use of the application and a progressive decline in activity during the week, resulting in almost zero activity on weekends. However, participants who followed the recommendations were able to perform the exercises with less effort, thus increasing their lean mass and improving their sleep quality. 

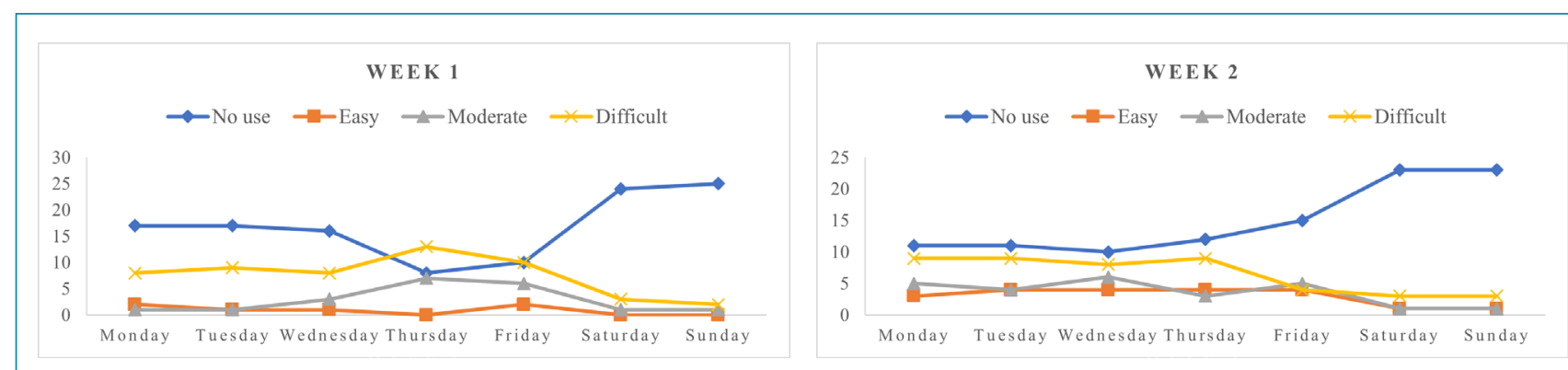

Mean \pm SD: 2.82 \pm 1.47 - Minimum 0 - Maximum 5 - Vertical axis: sample (n).
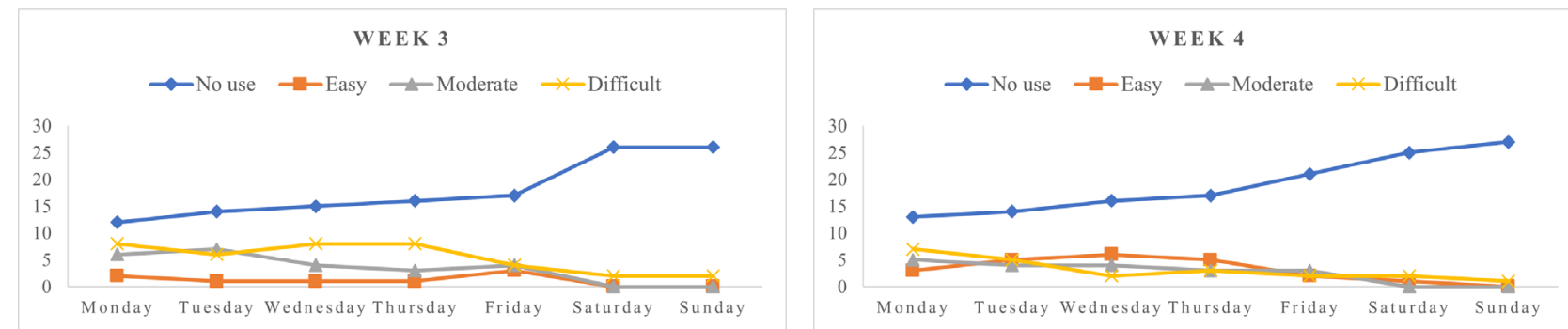

Mean $\pm S D: 2.50 \pm 2.17$ - Minimum 0 - Maximum 5-Vertical axis: sample (n).
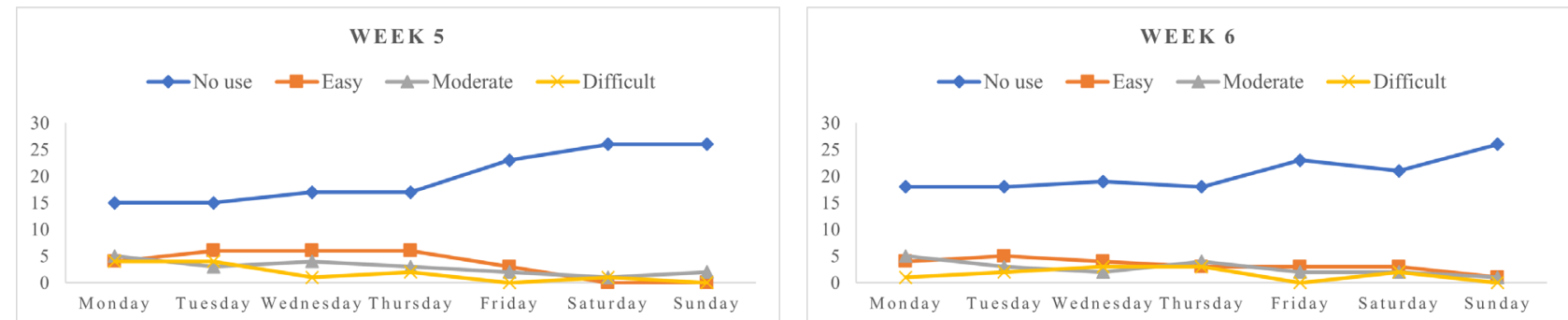

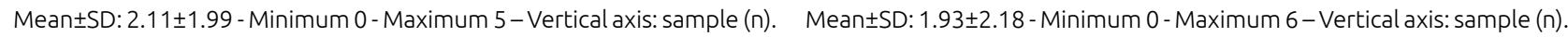

Figure 1. Daily perceived exertion ratings on the Borg Scale for 6 weeks.

Table 1. Comparison of body composition variables and total sleep time before and after using the mobile application.

\begin{tabular}{|c|c|c|c|}
\hline Variables & $\begin{array}{l}\text { Before using the mobile } \\
\text { application } \\
(\mathrm{n}=28)\end{array}$ & $\begin{array}{l}\text { After using the mobile } \\
\text { application } \\
(n=28)\end{array}$ & $p$-value ${ }^{a}$ \\
\hline Weight, kg & $66.67 \pm 14.75$ & $67.82 \pm 15.32$ & 0.004 \\
\hline Height, m & $1.49 \pm 0.10$ & $1.50 \pm 0.10$ & 0.001 \\
\hline Height z-score & $1.08 \pm 1.40$ & $1.06 \pm 1.38$ & 0.70 \\
\hline $\mathrm{BMI}, \mathrm{kg} / \mathrm{m}^{2}$ & $29.62 \pm 4.84$ & $29.80 \pm 5.11$ & 0.28 \\
\hline BMI z-score & $2.26 \pm 0.38$ & $2.25 \pm 0.41$ & 0.50 \\
\hline Waist circumference, $\mathrm{cm}$ & $86.99 \pm 9.40$ & $86.66 \pm 9.85$ & 0.65 \\
\hline Total fat mass, kg & $22.58 \pm 7.60$ & $22.57 \pm 7.48$ & 0.99 \\
\hline Total lean mass, kg & $44.08 \pm 8.20$ & $45.42 \pm 9.20$ & 0.007 \\
\hline Body fat percentage, \% & $33.16 \pm 5.38$ & $32.62 \pm 5.19$ & 0.31 \\
\hline Sleep time, hours/day & $9.51 \pm 1.21$ & $9.14 \pm 1.31$ & 0.26 \\
\hline
\end{tabular}

BMI: body mass index aPaired t-test. Data presented as mean \pm standard deviation. 
Table 2. Sleep quality before and after using the mobile application.

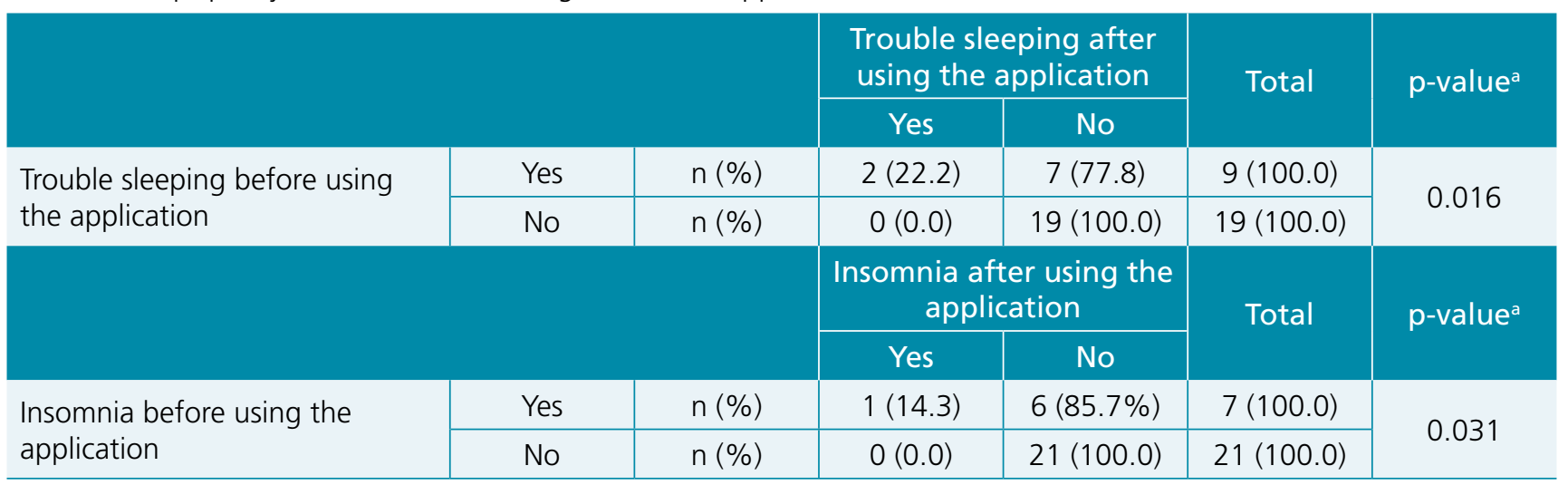

${ }^{a}$ Exact McNemar test.

The lower than recommended adherence to the application can be explained by the subjects' resistance to begin physical activities, the level of difficulty of the exercises, and a disturbed routine. The low level of interest may also be due to preferences for browsing or using other digital platforms ${ }^{11}$. It has been found that personalizing the frequency and intensity of the exercises, setting goals, knowing the benefits of exercise, and self-monitoring in real-time can promote adherence. Furthermore, encouragement from family members and healthcare professionals also plays an important role to promote children practicing physical activity ${ }^{5,11}$.

In this study, the decline in the use of the application on weekends suggests little family involvement in the development of healthy habits in children. Studies have shown a reduction in physical activity and an increase in sedentary behavior in obese parents and children on weekends, which indicates a difficulty in promoting games that are fun and stimulating for a child's healthy development ${ }^{12,13}$. It must be noted that family support is crucial for the development of healthy behaviors ${ }^{14}$.

The decrease in the level of difficulty and effort required to perform the proposed exercises can be explained by the continuous use of the application. Contact with the researchers via WhatsApp Messenger ${ }^{\circledR}$ may also have facilitated the patient's compliance with the recommended exercises. The study suggests that parents have a positive outlook towards messages that are clear and objective, and which contain practical instructions ${ }^{14}$.

As for body composition, there was a statistically significant increase in weight, height, and lean mass but no significant changes in BMI and BMI z-scores. The mean waist circumference, total fat mass, and body fat percentage were lower after the evaluations, but there were no statistical differences. We noted that the use of the application, even for just ten minutes a day for six weeks, generated positive results. The increase in physical activity led to an increase in energy expenditure and possibly reduced adiposity, since muscle activity is the primary form of energy consumption. However, the rapid increase in height may be related to weight gain, resulting in a decreased $\mathrm{BMI}^{15}$.

The average sleep time after the intervention was between nine and eleven hours, which is appropriate for this age group ${ }^{16}$. The patients had less trouble sleeping and less insomnia, which highlighted the effects of physical activity on sleep quality. Children who sleep less than nine hours a day and have poor sleep quality are more likely to increase their caloric intake and sedentary activities, which consequently leads to an increase in adiposity ${ }^{17}$. Nevertheless, physical activity can have a direct impact on these harmful habits and promote health benefits ${ }^{18}$.

Aside from regular contact with the researcher, the positive changes may also be related to consistent follow-up care at the multidisciplinary clinic. It has been found that lifestyle changes will have a positive impact only when they are consistently maintained in the long run, which indicates the importance of creating intervention strategies that can be incorporated gradually and engagingly $y^{19}$.

The strengths of the study included the use of a free and userfriendly mobile application to promote the health of patients in follow-up care, at a specialized outpatient clinic that was difficult to access, under remote supervision of researchers. It is important to note that during the pandemic, when social distancing was a safety measure, mobile applications emerged as a potential tool for healthcare professionals to help children remain active.

One of the limitations of the study was that the application was not specific to the age group of the study; it did not have specific goals, nor was it very playful. In addition, the sample size was small. These reasons may explain patients' low adherence to the use of the application and the progressive decline in its use during the six weeks of the intervention, especially on weekends. Those who followed the recommendations benefited by being able to perform the exercises with less effort, increasing their lean mass, and improving their sleep quality. We 
believe that playful applications with an interactive and "gamified" interface could generate better results for this age group.

\section{CONCLUSIONS}

The patients' use of the physical activity application led to an improvement in their quality of sleep and an increase in their lean mass, while there were no significant changes in BMI $\mathrm{z}$-scores, waist circumference, and body fat percentage.

\section{AUTHORS' CONTRIBUITIONS}

FF: Conceptualization, Data curation, Research, Methodology, Project management, Writing - review and editing. CCS: Data curation, Formal analysis, Software, Writing - review and editing. RTM: Formal Analysis, Visualization. MÂRGMA: Formal Analysis (Leadership), Supervision (Leadership), Writing - review and editing (Leadership). MPZ: Formal Analysis (Leadership), Financing acquisition (Leadership), Supervision (Leadership), Writing - review and editing (Leadership).

\section{REFERENCES}

1. Sousa P, Duarte E, Ferreira R, Esperança A, Frontini R, SantosRocha $\mathrm{R}$, et al. An mHealth intervention programme to promote healthy behaviours and prevent adolescent obesity (TeenPower): A study protocol. J Adv Nurs. 2019;75(3):68391. https://doi.org/10.1111/jan.13905

2. Barnett TA, Kelly AS, Young DR, Perry CK, Pratt CA, Edwards $N M$, et al. Sedentary behaviors in today's youth: approaches to the prevention and management of childhood obesity: a scientific statement from the American Heart Association. Circulation. 2018;138(11):e142-e59. https://doi.org/10.1161/ CIR.0000000000000591

3. Grady A, Yoong S, Sutherland R, Lee H, Nathan N, Wolfenden $\mathrm{L}$. Improving the public health impact of eHealth and mHealth interventions. Aust N Z J Public Health. 2018;42(2):118-9. https://doi.org/10.1111/1753-6405.12771

4. Direito A, Jiang Y, Whittaker R, Maddison R. Apps for improving fitness and increasing physical activity among young people: The AIMFIT pragmatic randomized controlled trial. J Med Internet Res. 2015;17(8):e210. https://doi.org/10.2196/ jmir.4568

5. Direito A, Carraça E, Rawstorn J, Whittaker R, Maddison R. mHealth Technologies to Influence Physical Activity and Sedentary Behaviors: Behavior Change Techniques, Systematic Review and Meta-Analysis of Randomized Controlled Trials. Ann Behav Med. 2017;51(2):226-39. https://doi.org/10.1007/ s12160-016-9846-0

6. Borg G. Perceived exertion as an indicator of somatic stress. Scand J Rehabil Med. 1970;2(2):92-8. PMID: 5523831

7. Norgan NG. A Review of: "Anthropometric Standardization Reference Manual ". Edited by Lohman TG, Roche AF, Martorell R. (Champaign, IL.: Human Kinetics Books, 1988). Ergonomics. 1988;31(10):1493-4. https://doi. org/10.1080/00140138808966796

8. Kuczmarski RJ, Ogden CL, Guo SS, Grummer-Strawn LM, Flegal KM, Mei Z, et al. 2000 CDC growth charts for the United States: methods and development. Vital Health Stat. 2002;(246):1-190. PMID: 12043359

9. Fryar $C D, G u$ Q, Ogden $C L$. Anthropometric reference data for children and adults: United states, 2007-2010. Vital Health Stat. 2012;(252):1-48. PMID: 25204692
10. Lukaski HC, Bolonchuk WW, Hall CB, Siders WA. Validation of tetrapolar bioelectrical impedance method to assess human body composition. J Appl Physiol (1985). 1986;60(4):1327-32. https://doi.org/10.1152/jappl.1986.60.4.1327

11. Lee J, Jeongeun K, Ahjung B, Meiling J, Meihua P, Kyungryeon K, et al. Application design for child obesity management based on users' preferences and needs. West J Nurs Res. 2020;42(5):35664. https://doi.org/10.1177/0193945919862797

12. Tremblay MS, Gray C, Babcock S, Barnes J, Bradstreet CC, Carr D, et al. Position statement on active outdoor play. Int J Environ Res Public Health. 2015;12(6):6475-505. https://doi. org/10.3390/ijerph120606475

13. Foster $C$, Moore JB, Singletary CR, Skelton JA. Physical activity and family-based obesity treatment: a review of expert recommendations on physical activity in youth. Clin Obes. 2018;8(1):68-79. https://doi.org/10.1111/cob.12230

14. Callender C, Thompson D. Text Messaging based obesity prevention program for parents of pre-adolescent African American Girls. Children (Basel). 2017;4(12):105. https://doi. org/10.3390/children4120105

15. Bichteler A, Gershoff ET. Identification of children's BMI trajectories and prediction from weight gain in infancy. Obesity (Silver Spring). 2018;26(6):1050-6. https://doi.org/10.1002/oby.22177

16. Paruthi S, Brooks LJ, D'Ambrosio C, Hall WA, Kotagal S, Lloyd RM, et al. Recommended amount of sleep for pediatric populations: a consensus statement of the American Academy of Sleep Medicine. J Clin Sleep Med. 2016;12(06):785-6. https://doi.org/10.5664/jcsm.5866

17. Fatima Y, Doi SAR, Mamun AA. Longitudinal impact of sleep on overweight and obesity in children and adolescents: a systematic review and bias-adjusted meta-analysis. Obes Rev. 2015;16(2):137-49. https://doi.org/10.1111/obr.12245

18. Saunders TJ, Gray CE, Poitras VJ, Chaput JP, Janssen I, Katzmarzyk PT, et al. Combinations of physical activity, sedentary behaviour and sleep: relationships with health indicators in school-aged children and youth. Appl Physiol Nutr Metab. 2016;41(6 Suppl 3):S283-93. https://doi.org/10.1139/apnm-2015-0626

19. Lobelo F, Muth ND, Hanson S, Nemeth BA. Physical activity assessment and counseling in pediatric clinical settings. Pediatrics. 2020;145(3):e20193992. https://doi.org/10.1542/peds.2019-3992 